\title{
Grid-Aware versus Grid-Agnostic Distribution System Control: A Method for Certifying Engineering Constraint Satisfaction
}

\author{
Daniel K. Molzahn \\ Argonne National Laboratory \\ dmolzahn@anl.gov
}

\author{
Line A. Roald \\ Los Alamos National Laboratory \\ roald@lanl.gov
}

\begin{abstract}
Growing penetrations of distributed energy resources (DERs) in distribution systems have motivated the design of controllers that leverage DER capabilities to achieve system-wide objectives. These controllers may be either grid-agnostic or grid-aware, depending on whether distribution network constraints are considered. Grid-agnostic controllers have the benefit of not requiring network models or system measurements, but may cause dangerous constraint violations. Rather than develop a specific controller, this paper considers the potential impacts of DER controllers with respect to network constraint violations. Specifically, this paper develops an optimization-based method to rigorously certify when any grid-agnostic controller can be applied without concern regarding network constraint violations, or, conversely, when grid-aware control may be needed to maintain distribution grid security. The proposed method uses convex optimization techniques to bound the impacts of load variability, given a subset of buses with voltage measurements and control. The method either provides a certificate for secure operation or identifies potentially critical constraints and the need for additional controllability. Numerical tests illustrate the ability to certify secure operation for different ranges of variability.
\end{abstract}

\section{Introduction}

Rapidly growing penetrations of distributed energy resources (DERs) such as solar PV generators, plug-in electric vehicles, demand response, and energy storage are affecting the design and operation of electric distribution systems [1-9]. Many research efforts have focused on the development of controllers that coordinate DERs in order to achieve system-wide objectives, such as frequency control [2,3], voltage control [10-12], system stability [3,13], and mitigating phase unbalance [14]. We divide these controllers into two categories. Grid-agnostic DER controllers do not consider the distribution system's engineering constraints. Instead, they focus on achieving a desired aggregated performance, such as tracking setpoints for active and reactive power, which might be sent from an entity that aims at balancing the grid [2,3], controlling the aggregate consumption across loads [15], or responding to market prices [16]. Conversely, grid-aware controllers actively manage DER outputs to avoid or mitigate violations of engineering constraints such as voltage limits $[11,12]$, prevent overloading of transformers, or defer investments $[4,17]$, sometimes in presence of load uncertainty [18].

These categories of controllers have inherent trade-offs between implementation complexity and their ability to handle grid constraints. Grid-agnostic controllers are often beneficial as they do not require a distribution system model or real-time measurements. They can hence be managed by entities other than the distribution grid operator such as the transmission system operator or a third-party DER aggregator, which may consolidate DER capabilities over a large geographical area. However, use of grid-agnostic controllers without further consideration of distribution grid constraints may aggravate power quality concerns and damage equipment. Conversely, while grid-aware controllers are designed to mitigate network problems, their requirements on information about individual loads and network models increase implementation complexity and might cause privacy concerns.

Distribution system operators hence need tools to understand under which circumstances grid-agnostic control can be applied without consequences for grid security and, conversely, when it is necessary to invest in the sensing, communication, and control infrastructure required to observe and mitigate constraint violations using grid-aware control. Existing approaches for analyzing this question, such as $[1,7,9]$, perform multiple simulations in order to study the risk of constraint violations associated with fluctuations from various grid-agnostic controllers and variable loads. These approaches provide valuable information 
regarding the impacts of DERs on distribution systems, but are limited to only considering a subset of possible operational scenarios.

Rather than simulating a select number of scenarios, this paper proposes a rigorous method to certify whether distribution system operation will remain secure in the face of a range of variability. We consider a set of buses with voltage measurements and control as well as specified ranges for the power injections at all buses which represent possible realizations of variable loads, renewable generators, and both grid-aware and grid-agnostic DER control. Our method either certifies that no combination of the power injections within these considered ranges will lead to constraint violations or identifies the possible need for additional measurements and controls to ensure security. These certificates help evaluate the security implications of adding new renewable generators and the application of grid-agnostic DER controllers.

The certificates for secure operation are obtained by solving a minimization or maximization problem for each constraint which bounds the impact of the variability. We employ convex relaxations of the $\mathrm{AC}$ power flow constraints to obtain valid but conservative bounds for the extreme values obtained from these problems. We use the QC relaxation [19-21], but other relaxations are also applicable [22].

We illustrate our method using the 56-bus system from [23], which is derived from the IEEE 123-bus test case. Our method enables us to certify security for various operating ranges, given a very limited number of buses with voltage measurements and control.

The main contributions of this paper are:

(i) A method to certify distribution grid security, i.e., no constraint violations can occur for any combination of power injections within certain ranges.

(ii) A principled approach to certify secure operations for a broader range of operational conditions, i.e., larger ranges of load variability, given the enforcement of a small number of voltage limits via grid-aware control.

(iii) Numerical simulations which demonstrate the benefits of the proposed method.

The remainder of this paper is organized as follows. Section 2 provides the problem setting, introduces notation, and describes the problem formulation and our solution method. Section 3 numerically illustrates the proposed method. Section 4 concludes the paper and discusses future work.

\section{Problem Description}

This paper develops a method for distribution grid operators to certify secure operation for a range of operational conditions, given limited measurement and

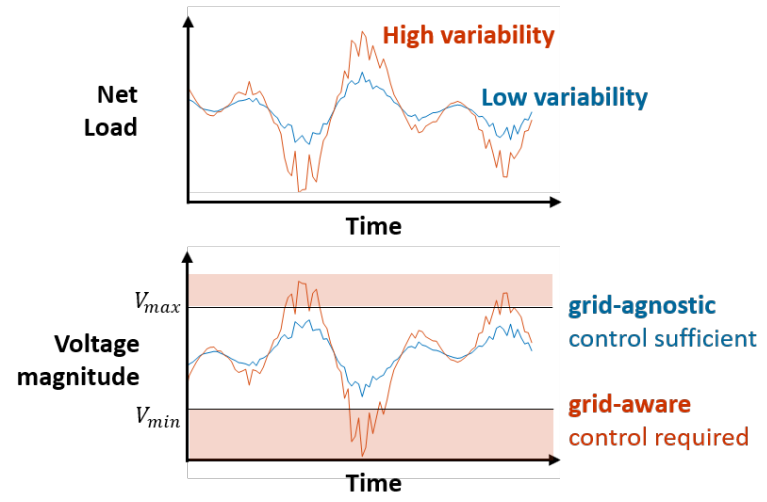

Figure 1. If not used cautiously, grid-agnostic DER control can increase the variability in distribution system load patterns and lead to constraint violations.

control capabilities. Secure operation in this context refers to the situation where all engineering limits are respected for a range of load variability, as represented by ranges of active and reactive power injections at each bus. The certificates for secure operations are obtained by solving a set of optimization problems. To state our problem of interest, we first describe the variability which may cause distribution grid constraint violations and then give our definitions of grid awareness and control. We next formalize this problem and describe our solution method.

We emphasize that we do not propose a new DER controller, but instead certify security for distribution grids with limited measurement and control capabilities, independent of the specific choice of DER controller. Also note that this paper focuses on enforcing voltage magnitude limits, which are typically of greatest concern in distribution grid operations [9]. A similar methodology could be applied to investigate, e.g., current flow or voltage unbalance. Finally, note that while this paper focuses on distribution systems, our method does not require typical distribution system characteristics such as a radial network topology. Thus, the developed algorithm can be applied to a broader class of systems (e.g., meshed distribution grids, transmission systems).

\subsection{Load Variability}

Load variability is defined as a range of net power injections at each bus,

$$
\underline{P}_{i} \leq P_{i} \leq \bar{P}_{i} \text { and } \underline{Q}_{i} \leq Q_{i} \leq \bar{Q}_{i}, \forall i \in \mathcal{N}
$$

where $P_{i}$ and $Q_{i}$ denote the net active and reactive power injections, and $\mathcal{N}$ is the set of buses. The power injection ranges $\underline{P}, \bar{P}$ and $\underline{Q}, \bar{Q}$ model different sources 
of variability:

- Loading pattern variability results from the traditional daily and seasonal consumption patterns that the distribution grid was designed to handle.

- Uncontrolled DER variability results from (uncontrolled) distributed energy sources such as rooftop PV or plug-in electric vehicle charging.

- Grid-agnostic or grid-aware DER control variability results from the changes in the DER behavior to meet certain objectives, in a manner that may be either agnostic or aware of grid constraints.

Note that we consider inherent variability (from uncontrollable loads) and variability induced by DER controllers (both exogenous control signals from grid-agnostic controllers and control response from grid-aware controllers) in the same manner. This allows us to treat all kinds of variability in a single framework, without considering further details about the source of the inherent variability and without specific assumptions about the type of DER control.

Our formulation and numerical results in this paper focus on ranges of power injections that represent fluctuations around a single nominal operating point. Practical applications of the proposed method could be extended to more detailed analyses that consider, e.g., multiple nominal operating points to represent typical daily and seasonal load patterns as well as more complicated variability representations than simple ranges of power injections (e.g., discrete changes due to loads that can only be switched on or off, $P-Q$ capability curves for certain types of DERs, and the power factor characteristics of variable loads). While more detailed variability characterizations will improve our method by ensuring that only realistic loading scenarios are included, the certificates for secure operation are only based on our consideration of the power injection ranges, and hence provide potentially conservative, but valid results.

\subsection{Grid Awareness and Control}

To mitigate adverse effects of load variability, the distribution system operator can harness DER flexibility via grid-aware control algorithms that specifically consider constraints such as voltage limits. However, distribution grids frequently have limited sensing, measurement, and communication capabilities, which means that the system operator only has limited awareness regarding the system state. Methods for guaranteeing secure operations while only requiring limited information about the system state are hence of interest to distribution grid operators.
This paper considers the following definitions:

(i) Grid awareness consists of measurements of nodal voltage magnitudes $V_{j}$ on a subset $\mathcal{V} \subseteq \mathcal{N}$ of the buses in the distribution grid.

(ii) Grid controllability is related to the operator's ability to control the voltage magnitudes $V_{j}$ at some or all of the buses $j \in \mathcal{V}$ to be within certain ranges $V_{j}, \tilde{V}_{j}$.

We assume that the voltage magnitude at the substation is always measured by the distribution system operator and that it is typically controlled to remain relatively constant (e.g., $\tilde{V}_{j} \approx V_{j} \approx 1$ p.u.). The set $\mathcal{V}$ therefore always contains at least one bus.

In some cases, the distribution system operator has access to measurements of the voltage magnitudes at additional buses throughout the system, and some or all of those voltage magnitudes may be controlled via changes to the DER power injections. In this case, we augment the set $\mathcal{V}$ to include these additional buses and consider their corresponding voltage magnitude ranges.

Note that we do not propose a specific DER control strategy in this paper. We merely assume that there exists a grid-aware DER control strategy which is able to maintain the voltage bounds $V_{j}, \tilde{V}_{j}$ at buses $j \in \mathcal{V}$ given the pre-specified ranges of power injections, as will be further described in Section 2.3.4.

\subsection{Certifying Secure Operations}

Given the load variability defined by the power injection ranges in (1) and the availability of voltage magnitude measurements and control at certain buses $\mathcal{V} \subseteq \mathcal{N}$, we investigate whether it is possible to violate any voltage magnitude constraints. Specifically, we ask whether there exists a combination of power injections within the specified ranges $\underline{P}, \bar{P}$ and $Q, \bar{Q}$ that would lead to voltage violations at any bus, given that the voltage magnitudes are kept within the specified variability ranges $V_{j}, \tilde{V}_{j}$ at the controllable buses $j \in$ $\mathcal{V}$. We next formulate an optimization problem which enables us rule out the existence of such a combination of power injections, hence certifying that operations will be secure for all considered load variability realizations.

2.3.1. Notation Consider a power system with sets of buses $\mathcal{N}$ and lines $\mathcal{L}$. Each bus $i \in \mathcal{N}$ has a complex voltage phasor $V_{i} \angle \theta_{i}$ and complex power injection $P_{i}+\mathbf{j} Q_{i}$, where $\mathbf{j}=\sqrt{-1}$. We consider a range of power injections at each bus defined by (1). The network admittance matrix is denoted $\mathbf{Y}=\mathbf{G}+\mathbf{j B}$. Voltage angle differences are denoted $\theta_{l m}=\theta_{l}-\theta_{m}$, $\forall(l, m) \in \mathcal{L}$. Subscript "ref" denotes the reference bus.

We consider three different types of voltage bounds:

(i) Upper and lower engineering limits on the voltage 
magnitudes, denoted as $V_{i}^{\max }$ and $V_{i}^{\text {min }}$, corresponding to the secure operating ranges which reflect power quality and safety requirements. ${ }^{1}$

(ii) Specified operational ranges for the voltage magnitudes are denoted by ${\underset{\sim}{j}}_{j} \leq V_{j} \leq \tilde{V}_{j}$ at the buses $j \in \mathcal{V}$ with available voltage measurements and control. These operational ranges are typically narrower than the engineering limits, i.e.,

$$
V_{j}^{\text {min }} \leq V_{j} \leq V_{j} \leq \tilde{V}_{j} \leq V_{j}^{\max }, \quad \forall j \in \mathcal{V}
$$

(iii) Maximum and minimum achievable values of the voltage magnitudes, given the ranges of power injections (1) and the voltage operating ranges (2), which are denoted as $\bar{V}_{i}$ and $\underline{V}_{i}, \forall i \in \mathcal{N}$. The maximum and minimum achievable voltage values typically differ from the engineering limits, with a wider or a narrower range depending on the system. We also denote the maximum and minimum achievable voltage angle differences as $\bar{\theta}_{l m}, \underline{\theta}_{l m}, \forall(l, m) \in \mathcal{L}$.

2.3.2. Optimization Problem Formulation To identify whether it is possible to violate any voltage constraints for a given range of load variability, we solve the following optimization problems that maximize and minimize the voltage magnitudes $V_{n}$ at each bus $n \in \mathcal{N}$ :

$$
\begin{array}{lr}
\underline{V}_{n}=\min _{P, Q, V, \theta} V_{n} \quad \text { or } \quad \bar{V}_{n}=\max _{P, Q, V, \theta} V_{n} \\
\text { subject to } \quad \forall i \in \mathcal{N}) & \forall k \in \mathcal{N} \backslash r e f, \\
\underline{P}_{k} \leq P_{k} \leq \bar{P}_{k}, & \forall k \in \mathcal{N} \backslash r e f, \\
\underline{Q}_{k} \leq Q_{k} \leq \bar{Q}_{k}, & \forall j \in \mathcal{V}, \\
V_{j} \leq V_{j} \leq \tilde{V}_{j}, & \\
P_{i}=V_{i} \sum_{k \in \mathcal{N}} V_{k}\left(\mathbf{G}_{i k} \cos \left(\theta_{i k}\right)+\mathbf{B}_{i k} \sin \left(\theta_{i k}\right)\right), \\
\begin{array}{lr}
Q_{i}=V_{i} \sum_{k \in \mathcal{N}} V_{k}\left(\mathbf{G}_{i k} \sin \left(\theta_{i k}\right)-\mathbf{B}_{i k} \cos \left(\theta_{i k}\right)\right), \\
\theta_{r e f}=0,
\end{array} \\
V_{l b} \leq V_{i} .
\end{array}
$$

The objective (3a) minimizes or maximizes the voltage magnitude achievable at a particular bus $n \in \mathcal{N}$. The constraints (3b)-(3c) correspond to the loads' specified power injections ranges. Note that the power injection at the reference bus (the substation) is unconstrained, i.e., the distribution grid does not have limitations on the power from the transmission grid.

The voltage magnitude limits in $(3 \mathrm{~d})$ correspond to

\footnotetext{
${ }^{1}$ Although engineering limits on the current flows, $I_{l m}^{\max }$, $\forall(l, m) \in \mathcal{L}$, could also be included in the analysis, these limits are not considered in this paper for brevity.
}

the specified operational ranges. These constraints only include buses where we are able to measure and control the voltage magnitudes. We do not enforce voltage magnitude constraints for other buses $i \notin \mathcal{V}$ since we have no means of identifying or mitigating constraint violations at those buses in real time. Instead, we rely on the optimization problem (3) to provide the minimum and maximum achievable voltage magnitude values.

Constraints $(3 \mathrm{e})-(3 \mathrm{~h})$ model the AC power flow physics. Specifically, (3e) and (3f) enforce active and reactive power balance at each bus and $(3 \mathrm{~g})$ sets the voltage angle at the reference bus to zero. The technical condition $(3 \mathrm{~h}$ ) forces all voltage magnitudes to be greater than a specified scalar parameter $V_{l b}$ that is chosen to avoid undesirable "low-voltage" power flow solutions [24]. The lower bound $V_{l b}$ is selected to be significantly below any practical operating voltage, e.g., $V_{l b}=0.7$ p.u., such that all practically meaningful power flow solutions will be considered by the optimization problem (3).

Note that our method relies on assumptions regarding AC power flow feasibility. Specifically, we assume the existence of a high-voltage power flow solution for any combination of the power injections (i.e., the distribution grid is steady-state stable for all power injections within the specified ranges) and that all potential low-voltage solutions are precluded by (3h). While these are mild assumptions for typical operating ranges, our ongoing work aims to leverage recently developed "convex restriction" techniques $[25,26]$ to reduce our dependence on these assumptions.

Although we focus on voltage magnitude constraints, related problems could be formulated in order to consider other relevant engineering limits if required (e.g., current flow limits).

\subsubsection{Successful Certificate for Secure Operations} If the minimum and maximum achievable voltage magnitudes obtained from (3) are within the specified engineering limits $V^{\min }, V^{\max }$ on all buses, i.e.,

$$
V_{i}^{\min } \leq \underline{V}_{i} \text { and } \bar{V}_{i} \leq V_{i}^{\max }, \forall i \in \mathcal{N}
$$

then the power flow solutions associated with all possible power injections within the specified ranges (1) will satisfy the engineering limits on the voltage magnitudes. This result guarantees security with respect to all voltage magnitude limits as long as the total load variability remains within the specified ranges (1) and the voltage magnitudes are kept within $V_{j}, \tilde{V}_{j}$ at the subset of controllable buses $j \in \mathcal{V}$.

2.3.4. Mitigating Potentially Insecure Operations The extreme achievable voltages $\underline{V}_{i}$ and $\bar{V}_{i}$ depend 
on the considered range of load variability (1) and our ability to measure and control the voltage magnitudes at the specified buses (2). As the range of possible power injections increases, we will observe more significant changes in the voltage magnitudes. For sufficiently large variability ranges, some achievable voltages will eventually exceed the voltage limits, hence violating (4) and resulting in an inability to certify secure operations without additional grid awareness and control.

As a countermeasure to increasing variability, we may impose tighter operational ranges as this also decreases the ranges of voltage magnitudes that are achievable at other buses. This corresponds to either decreasing the operational range $V_{j}, \tilde{V}_{j}$ for a bus $j \in \mathcal{V}$ or adding buses to the set $\mathcal{V}$. From a practical perspective, the former corresponds to investing in more control capability at a bus with existing measurement and control, while the latter would require investments in both additional sensors and control capabilities.

Restricting the range of operation considered in (3) results in less extreme values for $V_{i}$ and $\bar{V}_{i}$. If the resulting values for $\underline{V}_{i}$ and $\bar{V}_{i}$ satisfy (4), then we successfully certify that all voltage magnitudes will remain within their engineering limits, as long as the specified limits on the voltage magnitudes are satisfied and the loads remain within the specified ranges (i.e., (3b)-(3d) are satisfied). If the resulting values for $\underline{V}_{i}$ and $\bar{V}_{i}$ do not satisfy (4), we continue to tighten the voltage magnitude ranges or further augment the set $\mathcal{V}$.

Note that we do not show how to formulate controllers that maintain the voltage magnitudes within the operational ranges $V_{j}, \tilde{V}_{j}, \forall j \in \mathcal{V}$, or even guarantee that such a controller exists. Rather, we certify that any grid-aware controller that enforces these additional constraints by modifying the power injections of DERs (within the load ranges specified by (1)) is sufficient to ensure that the remaining (unobserved) voltage magnitudes will be within the secure operating range $V^{\min }, V^{\max }$. The grid-aware controllers used to regulate the voltage can be complex optimization-based centralized controllers or simple local control loops. We refer the reader to existing literature, e.g., [8,10-12], for information about relevant grid-aware controllers.

Choosing appropriate locations $\mathcal{V}$ and operating ranges $V_{j}, \tilde{V}_{j}$ for the controllable buses should be done while accounting for the locations of the controllable resources, existing communication and sensing infrastructures, and other grid-specific characteristics. While out of scope for this paper, evaluating the possible choices of locations is part of our ongoing work. For our further discussions, we assume that a few candidate sets of buses $\mathcal{V}$ and operational ranges $V_{j}, \tilde{V}_{j}$ are known a-priori, and investigate the impact of controlling the voltages at these buses.

\subsection{Obtaining Valid Bounds on the Maximum and Minimum Achievable Values}

To evaluate the condition for secure operation (4), we solve the optimization problems (3) to obtain the extreme achievable values of the voltage magnitudes, $\underline{V}_{i}$ and $\bar{V}_{i}$, for all buses in the system. Reliably computing these extreme values is challenging due to the $\mathrm{AC}$ power flow constraints (3e), (3f). The non-convexity of these constraints implies that there might exist several locally optimal solutions to (3), where some solutions have more or less extreme values of the voltage magnitudes than others. If we find the wrong locally optimal solution, we might underestimate the influence of the load variability and falsely certify that operations will remain secure. Instead, we would like to find globally optimal solutions to the optimization problems (3), which allow us to rigorously guarantee that the condition for secure operations (4) holds.

Certifiably finding globally optimal solutions for optimization problems with AC power flow constraints is an active research topic [22], and is generally difficult [27, 28]. Instead of directly searching for the globally optimal solution to (3), we leverage recently developed convex relaxation techniques that replace non-convex constraints with convex outer approximations to obtain a more tractable formulation. Since a convex relaxation extends the feasible space of the optimization problem, the objective value of the relaxed problem provides a lower (in case of minimization) or upper (in case of maximization) bound on the true objective value. Specifically, if we denote the globally optimal objective values of the original problems (3) by $\underline{V}^{\text {true }}, \bar{V}^{\text {true }}$ and the globally optimal objective value of a convex relaxation of (3) by $\underline{V}^{\text {relax }}, \bar{V}^{\text {relax }}$, we have the following relations:

$$
\underline{V}^{\text {relax }} \leq \underline{V}^{\text {true }} \text { and } \bar{V}^{\text {true }} \leq \bar{V}^{\text {relax }} .
$$

Thus, the values obtained from convex relaxations are conservative bounds on the extreme achievable voltage magnitudes. We can therefore safely replace (3) with a convex relaxation of (3), which is useful since solvers that provide globally optimal solutions to convex optimization problems are readily available. ${ }^{2}$

2.4.1. Choice of Convex Relaxation There are many choices for convex relaxations of the AC power flow equations [22]. We use two main criteria to choose an appropriate relaxation: (i) tightness, which refers to how well the relaxation approximates the AC

\footnotetext{
${ }^{2}$ The decision variables $P_{i}, Q_{i}, \theta_{i}$ and $V_{i}$ from a convex relaxation may not satisfy the AC power flow equations (3e), (3f). However, this is not important when we are trying to evaluate the extreme achievable values of the voltage magnitudes since we never directly use decision variables, but only the objective values $\underline{V}, \bar{V}$.
} 


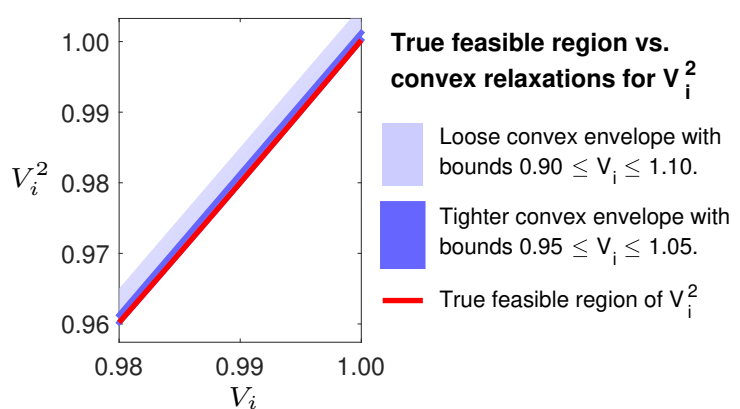

Figure 2. Convex relaxation for squared voltage magnitudes used in the $Q C$ relaxation.

power flow equations and thus how close the relaxation's objective value is to the true objective value, and (ii) the computational effort required to solve the relaxation.

We employ the QC relaxation [19-21] due to its computational tractability and tightness. The QC relaxation is derived from the power flow equations with voltage phasors represented in polar coordinates. The sine and cosine functions and the squared voltage magnitudes in (3e) and (3f) are replaced by a new set of lifted variables that are enclosed in corresponding convex envelopes. As an illustrative example, Figure 2 shows the feasible regions corresponding to the original non-convex constraint as well as convex envelopes for the squared voltage magnitudes, $V_{i}^{2}{ }^{3}$ The feasible regions of two different convex envelopes are shown, each corresponding to different variable bounds on $V_{i}$. A closer matching between the convex feasible region (in blue) with the original constraint (in red) results in better performance. A relaxation using envelopes constructed with tighter variable bounds will hence provide a better bound on the objective value of the original non-convex problem, a property exploited by our method. Note that the envelopes in the QC relaxation are formulated as linear and second-order cone constraints, resulting in problems that can be solved via efficient commercial optimization packages.

2.4.2. Variable Bounds in the QC Relaxation The convex envelopes for the trigonometric functions, squared voltage magnitudes, and variable products employed in the QC relaxation are constructed using upper and lower bounds on the voltage magnitudes and the angle differences between connected buses,

$$
\underline{V}_{i} \leq V_{i} \leq \bar{V}_{i}, \quad \forall i \in \mathcal{N}
$$

${ }^{3}$ The envelope for $V_{i}^{2} \quad$ is $\quad\left\{U_{i} \mid U_{i} \geq V_{i}^{2} \quad\right.$ and $\left.U_{i} \leq\left(V_{i}^{\min }+V_{i}^{\max }\right) V_{i}-V_{i}^{\min } V_{i}^{\max }\right\}$, where $U_{i}$ is a lifted variable representing the squared voltage magnitude at bus $i$. Envelopes for the other terms in (3e) and (3f) are given in [19-21].

$$
\underline{\theta}_{l m} \leq \theta_{l m} \leq \bar{\theta}_{l m}, \quad \forall(l, m) \in \mathcal{L} .
$$

Since the optimization problem (3) does not include constraints on all voltage magnitudes and also does not include limits on the angle differences, we need to specify the bounds $\underline{V}_{i}, \bar{V}_{i}, \underline{\theta}_{l m}$, and $\bar{\theta}_{l m}$ in order to construct the relaxations. ${ }^{4}$ In order to avoid restricting the relaxations' feasible spaces, the variable bounds on the voltage magnitudes and angle differences are chosen to be well outside of typical operational ranges for distribution systems, e.g.,

$$
\begin{aligned}
\underline{V}_{i} & =0.7 \text { p.u. }, & \underline{\theta}_{l m} & =-30^{\circ}, \\
\bar{V}_{i} & =1.3 \text { p.u., } & \bar{\theta}_{l m} & =30^{\circ} .
\end{aligned}
$$

While we initialize our relaxation with the large variable bounds in (8), we subsequently tighten the variable bounds (i.e., reduce the maximum and increase the minimum bounds on the variables $V_{i}$ and $\theta_{l m}$ ) using a so-called bound tightening algorithm. This improves the tightness of the QC relaxation, which again leads to less conservative bounds on the extreme values $\underline{V}_{i}$ and $\bar{V}_{i}$. Bound tightening algorithms have previously been shown to be very effective for improving relaxations of optimal power flow (OPF) problems [20,31,32] and have also been used to solve robust OPF problems [33].

2.4.3. Bound Tightening Algorithm As shown in Algorithm 1, the bound tightening algorithm improves the initially specified bounds (8) by iteratively solving optimization problems that maximize and minimize the voltage magnitudes and angle differences, tightening the variable bounds, and then again solving the optimization problems. This procedure is repeated until the variable bounds do not improve between iterations.

The iterations of Algorithm 1 solve problems that are similar to (3) for each $n \in \mathcal{N}$ and $(g, h) \in \mathcal{L}$ :

$$
\begin{aligned}
\underline{V}_{n}^{\star}=\min _{P, Q, V, \theta} V_{n} \quad \text { or } \quad \bar{V}_{n}^{\star}=\max _{P, Q, V, \theta} V_{n} \text { or } \\
\underline{\theta}_{g h}^{\star}=\min _{P, Q, V, \theta} \theta_{g h} \quad \text { or } \quad \bar{\theta}_{g h}^{\star}=\max _{P, Q, V, \theta} \theta_{g h} \\
\text { subject to } \quad(\forall i \in \mathcal{N}, \forall(l, m) \in \mathcal{L}) \\
\quad \underline{V}_{i} \leq V_{i} \leq \bar{V}_{i} \\
\underline{\theta}_{l m} \leq \theta_{l m} \leq \bar{\theta}_{l m} \\
\quad \text { QC relaxation of }(3 \mathrm{e})-(3 \mathrm{~h}) .
\end{aligned}
$$

The main difference with respect to (3) is that (9) uses a convex relaxation of the power flow constraints

\footnotetext{
${ }^{4}$ Note that the need to define variable bounds is specific to certain relaxations, such as the QC relaxation. Other relaxations, such as those based on moment/sum-of-squares hierarchies [29,30], do not require the initial specification of these bounds.
} 


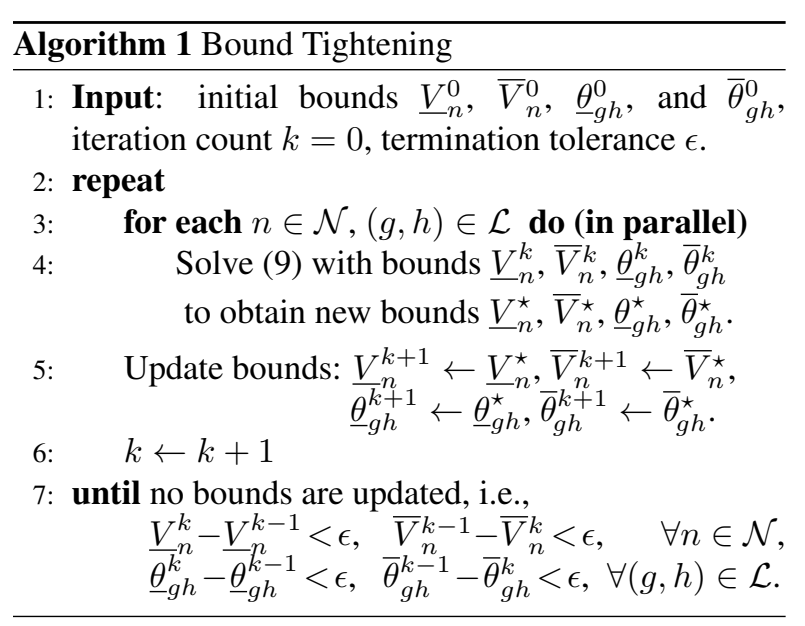

and includes maximization and minimization problems for the angle differences. Moreover, (9) includes (9b) and (9c) which bound the voltage magnitudes and angle differences based on the corresponding extreme achievable values, which would be redundant in (3). These bounds are initialized by (8) and tightened in subsequent iterations of the bound tightening algorithm. We emphasize again that the engineering limits $V_{i}^{\text {min }} \leq$ $V_{i} \leq V_{i}^{\max }$ are not enforced in (9) in order to allow for the possibility that some combination of power injections may result in unacceptable voltages.

The voltage magnitude bounds $\underline{V}_{i}$ and $\bar{V}_{i}$ resulting from Algorithm 1 are used to check whether the sufficient condition for secure operation (4) is satisfied. If not, we add additional measurements and controllability as described in Section 2.3.4 and repeat the analysis.

\section{Numerical Results}

We implement the QC relaxation and bound tightening algorithm based on [19-21] using YALMIP [34] in Matlab 2016a. The QC relaxations are solved using Mosek v8.0. All results shown in this paper are based on the solutions obtained via the QC relaxation and the bound tightening algorithm.

The remainder of this section illustrates the proposed method using the 56-bus radial test system in [23], which is a balanced single-phase system derived from the IEEE 123-bus system. The one-line diagram for the 56-bus system is shown in Figure 3(b). We define load variability as a percentage of the nominal loading and assume 1.0 p.u. voltage magnitude at the substation.

We run two different tests. First, we investigate how the bounds on the maximum and minimum voltage magnitudes at each bus change with an increasing range of load variability while assuming that the substation voltage is the only observed and controlled voltage magnitude in the distribution system. Second, we look into how enforcing voltage magnitude constraints on additional buses in the system can help improve the voltage profile throughout the distribution grid.

\subsection{Impacts of Increasing Load Variability}

Figure 3(a) shows the minimum and maximum achievable voltage magnitudes, $\underline{V}_{i}$ and $\bar{V}_{i}$, resulting from Algorithm 1 considering different amounts of load variability. The black dashed line in Figure 3(a) represents the baseline voltage magnitudes corresponding to the $\mathrm{AC}$ power flow solution at the nominal operating point (without variability). The buses are ordered by their baseline voltage magnitudes, decreasing from left to right. The shaded blue areas represent the ranges of voltage magnitudes as the load variability increases from $0 \%$ to $50 \%$, where each black line indicates an increase of $10 \%$. We consider voltage magnitudes below 0.90 p.u. to be unacceptable (i.e., $V^{\text {min }}=0.90$ p.u.), which corresponds to the red region. Figure 3(b) shows the system's one-line diagram with colors indicating our obtained lower bound on the achievable voltage magnitudes, $\underline{V}_{i}$, considering $50 \%$ load variability.

The bounds on the extreme achievable voltages increase with greater load variability. For load variability that exceeds approximately $10 \%$, we cannot certify secure operation since the minimum voltage magnitudes at several of the buses do not satisfy (4).

Remark: Conservativeness of the Convex Relaxation In Figure 3(a), the darkest blue region corresponds to the difference between the maximum and minimum achievable voltage magnitudes with $0 \%$ load variability, as bounded by the convex relaxation. While the maximum achievable voltage magnitudes are essentially the same as the baseline voltages obtained by solving the $\mathrm{AC}$ power flow equations, the bounds on the minimum achievable voltages from the convex relaxation are significantly lower than the baseline voltages. This can be attributed to the fact that the convex relaxation exploits inaccuracies in the outer approximation of the AC power flow constraints (3e), (3f) to artificially achieve lower voltage magnitudes. Tightening the relaxation via improvements such as those in [22, 29$31,35]$ may lead to less conservative results. We leave implementations of such improvements to future work.

\subsection{Impacts of Additional Grid Awareness and Control}

Since we are not able to certify secure operations for cases where the load variability exceeds $10 \%$, we introduce additional buses with voltage measurements 


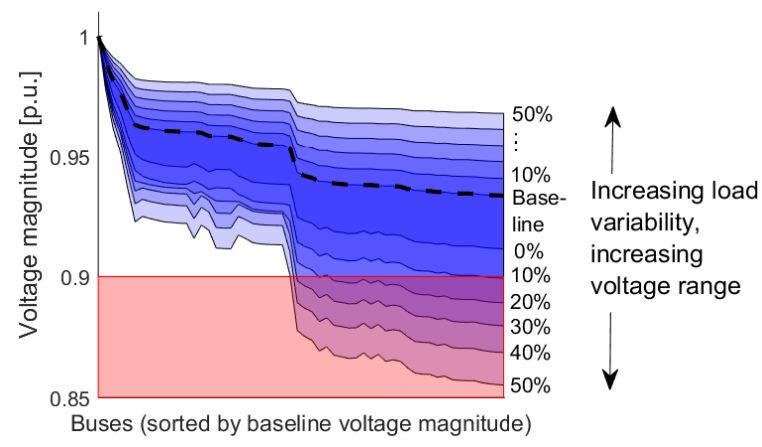

(a) Lower bounds on the achievable voltages, $\underline{V}_{i}$, for different amounts of load variability.

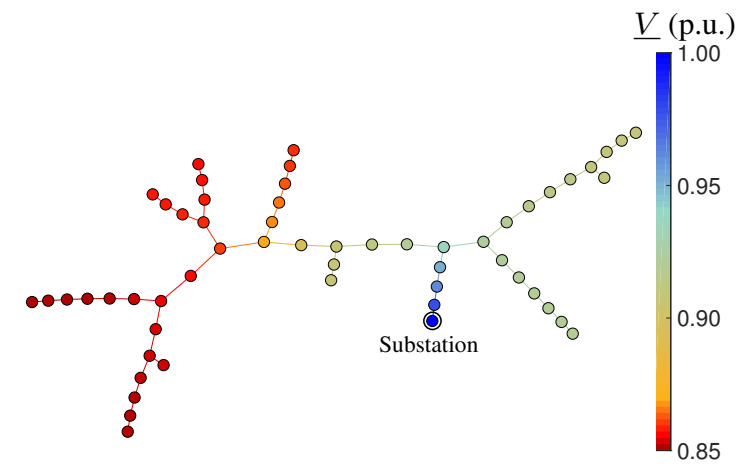

(b) One-line diagram for the 56-bus system. The colors denote the lower bounds on the achievable voltages, $\underline{V}_{i}$, for $50 \%$ load variability.

Figure 3. Voltage control only at the substation.

and control as described in Section 2.3.4. We choose to investigate the case with $50 \%$ load variability.

To find potential candidates for additional control, we study the voltage profile in the considered distribution grid. Figure 3(b) shows the minimum achievable voltage magnitudes without any control except at the substation. Unsurprisingly, the voltage magnitudes in the most remote parts of grid (i.e., the lower left part) have the lowest achievable voltages.

We first investigate the effect of monitoring and controlling the voltage at one of the leaf buses, bus 32, where the voltage magnitude is lowest. Formally, we augment $\mathcal{V}$ with bus 32 and impose $V_{32} \leq V_{32} \leq \tilde{V}_{32}$. Figure 4(a) shows the results of enforcing different values for $V_{32}$ and $\tilde{V}_{32}$. Choosing $\underset{\tilde{V}}{V_{32}}=0.90$ p.u. (denoted by the red triangle) and $\tilde{V}_{32}=1.10$ p.u. significantly reduces the potential size of voltage violations not only at bus 32 , but also throughout the system. By enforcing slightly tighter limits of $\underline{V}_{32}=0.905$ p.u. and $\tilde{V}_{32}=1.095$ p.u., we can guarantee secure operations for the entire system. The corresponding lower bounds on the voltages are illustrated in Figure 4(b).

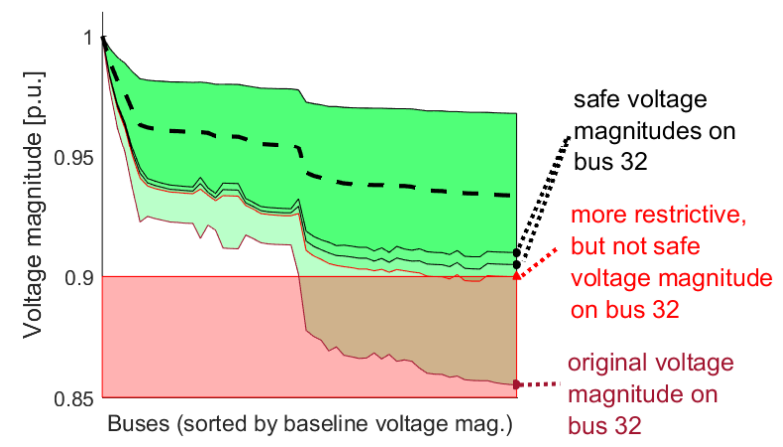

(a) Impacts of varying the voltage controllability at bus 32 with respect to the lower bounds on the achievable voltages, $\underline{V}_{i}$, for $50 \%$ load variability.

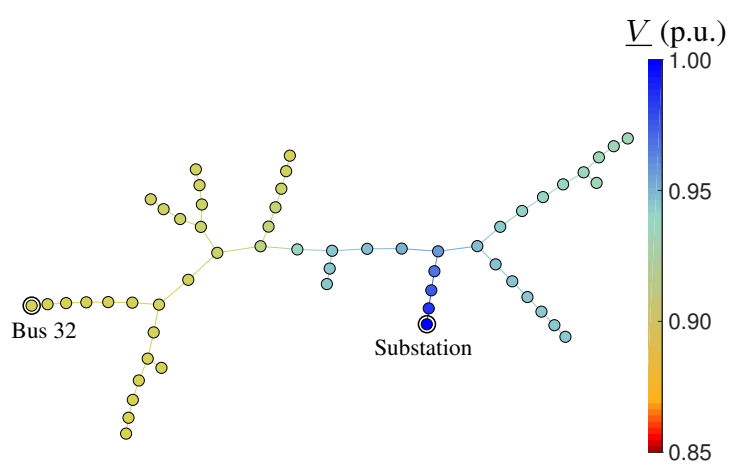

(b) One-line diagram for 56-bus system with $0.905 \leq V_{32} \leq 1.095$. The colors denote the lower bounds on the achievable voltages, $\underline{V}_{i}$, considering $50 \%$ load variability.

Figure 4. Voltage control at both the substation and bus 32 , considering $50 \%$ load variability.

Controlling the voltage magnitude at a leaf bus such as bus 32 might be inconvenient for a number of reasons. For example, the bus might be at the far end of a long feeder, making installation of communications difficult, or there might be limited controllability available at this bus. Therefore, we instead consider a different bus, bus 11 , as an alternative. The effect of controlling the voltage magnitude and enforcing different lower voltage limits at bus 11 is shown in Figure 5(a). As for bus 32 , we start by enforcing $\underline{\sim}_{11}=0.90$ p.u. and $\tilde{V}_{11}=1.10$ and then successively tighten the lower limit until we are able to certify secure operations. Enforcing $V_{11}=0.92$ p.u. and $\tilde{V}_{11}=1.08$ p.u. enables certification of secure operation for the entire system. The corresponding lower bounds on the voltages are illustrated in Figure 5(b).

These results show that it is possible to guarantee secure operations for the overall distribution grid by monitoring and controlling the voltage magnitudes at a subset of the buses. They also demonstrate that there are several options for guaranteeing secure operations. 


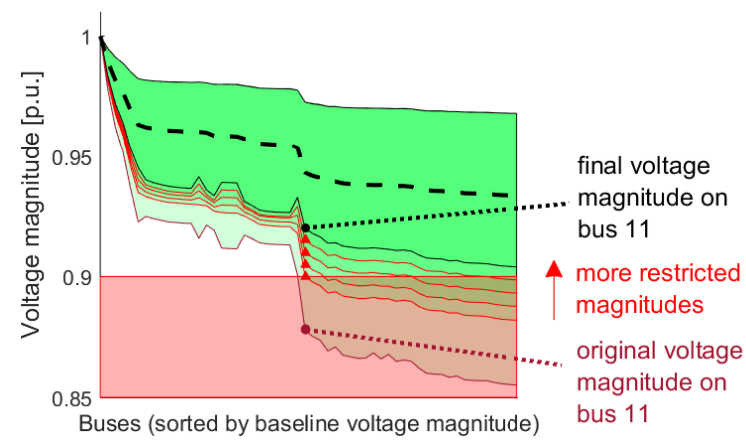

(a) Impacts of varying the voltage controllability at bus 11 with respect to the lower bounds on the achievable voltages, $V_{i}$, for $50 \%$ load variability.

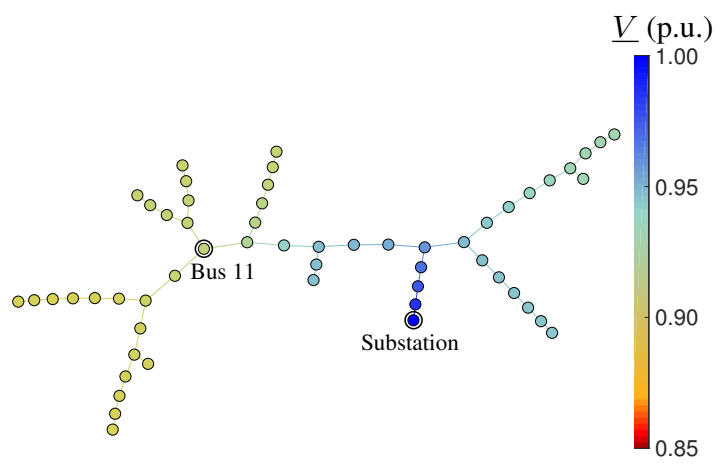

(b) One-line diagram for 56-bus system with $0.92 \leq V_{11} \leq 1.08$. The colors denote the lower bounds on the achievable voltages, $\underline{V}_{i}$, for $50 \%$ load variability.

Figure 5. Voltage control at both the substation and bus 11 , considering $50 \%$ load variability

This raises the question of how to choose the best combination of controllable buses $\mathcal{V}$ and allowable voltage ranges $\underline{V}_{j}$ and $\tilde{V}_{j}$. Developing methods to identify an optimal combination of measurements and control is an interesting aspect of future work.

Note that the operator does not necessarily have to continuously control the voltage magnitudes at the designated buses, but can instead use the measurements as an alert. In other words, the operator may wait until the measured voltage $V_{j}$ for some bus $j \in \mathcal{V}$ moves out of the range $\left[V_{j}, \tilde{V}_{j}\right]$ within which secure operation is guaranteed and then take some corrective action.

\section{Conclusions and Future Work}

Grid-agnostic DER controllers have advantages in terms of limited requirements on system model accuracy, information sharing, and real-time communication and sensing. However, the use of grid-agnostic controllers without careful consideration and analysis may aggravate violations of constraints such as limits on voltage magnitudes, particularly in systems that are already impacted by load fluctuations and variability from renewable generation. With the goal of facilitating the use of grid-agnostic DER control, this paper has proposed a method for certifying secure operations. Given specified ranges of load variability and a limited set of buses where voltages are measured and controlled, our security certificate guarantees that the voltage magnitudes at all buses will remain within an acceptable range. Using convex relaxations and a bound tightening algorithm, the proposed method computes bounds on the extreme voltage magnitudes achievable for any choice of power injections within specified ranges. If these extreme values are within the engineering limits on the voltage magnitudes, then no voltage magnitude constraint violations can occur.

For cases where we cannot certify engineering constraint feasibility for all considered power injections, we propose to augment the set of controllable buses or reduce the admissible voltage range at the already controllable buses. Executing our method with these limits can enable certification that grid-agnostic DER control is appropriate, provided that the distribution system operator is able to apply grid-aware control to enforce these limits.

Our ongoing work includes a variety of extensions to the current method. First, since unbalanced three-phase network models are more realistic representations of typical distribution systems, we are extending our method to consider the unbalanced three-phase AC power flow equations. We are also looking at including more granular models of the net load variability in our model (such as power factor constraints on controllable loads and DER capability curves). Further, we are applying recent improvements to tighten the convex relaxation in order to reduce the conservativeness of our results [22, 29-31,35]. Finally, we are developing more systematic methods for locating where to impose appropriately restrictive voltage constraints (i.e., the choice of buses in $\mathcal{V}$ and limits $V_{i}, \tilde{V}_{i}$ in (3d)) in order to securely apply grid-agnostic controllers without overly limiting DER capabilities.

\section{References}

[1] J. A. Taylor, A. Maitra, M. Alexander, D. Brooks, and M. Duvall, "Evaluations of plug-in electric vehicle distribution system impacts," in IEEE PES General Meeting, July 2010.

[2] D. S. Callaway and I. A. Hiskens, "Achieving controllability of electric loads," Proc. IEEE, vol. 99, no. 1, pp. 184-199, 2011.

[3] J. L. Mathieu, S. Koch, and D. S. Callaway, "State estimation and control of electric loads to manage real-time energy imbalance," IEEE Trans. Power Syst., vol. 28, no. 1, pp. 430-440, 2013.

[4] F. Oldewurtel, T. Borsche, M. Bucher, P. Fortenbacher, 
M. Gonzalez Vaya, T. Haring, J. L. Mathieu, O. Megel, E. Vrettos, and G. Andersson, "A framework for and assessment of demand response and energy storage in power systems," in IREP Symp.-IX, Aug. 2013.

[5] J. C. Mukherjee and A. Gupta, "A review of charge scheduling of electric vehicles in smart grid," IEEE Syst. J., vol. 9, no. 4, pp. 1541-1553, 2015.

[6] J. S. Vardakas, N. Zorba, and C. V. Verikoukis, "A survey on demand response programs in smart grids: Pricing methods and optimization algorithms," IEEE Commun. Surveys \& Tutorials, vol. 17, no. 1, pp. 152-178, 2015.

[7] S. C. Ross, G. Vuylsteke, and J. L. Mathieu, "Effects of load control for real-time energy balancing on distribution network constraints," in IEEE Manchester PowerTech, June 2017, pp. 1-6.

[8] D. K. Molzahn, F. Dörfler, H. Sandberg, S. H. Low, S. Chakrabarti, R. Baldick, and J. Lavaei, "A survey of distributed optimization and control algorithms for electric power systems," IEEE Trans. Smart Grid, vol. 8, no. 6, pp. 2939-2940, Nov. 2017.

[9] S. C. Ross, G. Vuylsteke, and J. L. Mathieu, "Effects of load-based frequency regulation on distribution network operation," arXiv:1807.03225, July 2018.

[10] K. E. Antoniadou-Plytaria, I. N. Kouveliotis-Lysikatos, P. S. Georgilakis, and N. D. Hatziargyriou, "Distributed and decentralized voltage control of smart distribution networks: Models, methods, and future research," IEEE Trans. Smart Grid, vol. 8, no. 6, pp. 2999-3008, Nov. 2017.

[11] E. Dall'Anese and A. Simonetto, "Optimal power flow pursuit," IEEE Trans. Smart Grid, vol. 9, no. 2, pp. 942-952, Mar. 2018.

[12] K. Baker, A. Bernstein, E. DallAnese, and C. Zhao, "Network-cognizant voltage droop control for distribution grids," IEEE Trans. Power Syst., vol. 33, no. 2, pp. 2098-2108, Mar. 2018.

[13] M. Yao, J. L. Mathieu, and D. K. Molzahn, "Using demand response to improve power system voltage stability margins," in IEEE Manchester PowerTech, June 2017.

[14] F. Nejabatkhah and Y. W. Li, "Flexible unbalanced compensation of three-phase distribution system using single-phase distributed generation inverters," to appear in IEEE Trans. Smart Grid, 2018.

[15] E. Vrettos, F. Oldewurtel, M. Vasirani, and G. Andersson, "Centralized and decentralized balance group optimization in electricity markets with demand response," in IEEE Grenoble PowerTech, June 2013.

[16] A. De Paola, D. Angeli, and G. Strbac, "Price-based schemes for distributed coordination of flexible demand in the electricity market," IEEE Trans. Smart Grid, vol. 8, no. 6, pp. 3104-3116, Nov. 2017.

[17] R. Tonkoski, L. A. C. Lopes, and T. H. M. El-Fouly, "Coordinated active power curtailment of grid connected PV inverters for overvoltage prevention," IEEE Trans. Sust. Energy, vol. 2, no. 2, pp. 139-147, Apr. 2011.

[18] S. Karagiannopoulos, L. Roald, P. Aristidou, and G. Hug, "Operational planning of active distribution grids under uncertainty," in IREP Symp.-X, Aug. 2017.

[19] C. Coffrin, H. L. Hijazi, and P. Van Hentenryck, "The QC relaxation: A theoretical and computational study on optimal power flow," IEEE Trans. Power Syst., vol. 31, no. 4, pp. 3008-3018, 2016.
[20] C. Coffrin, H. L. Hijazi, and P. Van Hentenryck, "Strengthening the SDP relaxation of AC power flows with convex envelopes, bound tightening, and valid inequalities," IEEE Trans. Power Syst., vol. 32, no. 5, pp. 3549-3558, Sept. 2017.

[21] K. Bestuzheva, H. L. Hijazi, and C. Coffrin, "Convex relaxations for quadratic on/off constraints and applications to optimal transmission switching," Preprint: http://www.optimization-online.org/DB_FILE/ 2016/07/5565.pdf, 2016.

[22] D. K. Molzahn and I. A. Hiskens, "A survey of relaxations and approximations of the power flow equations," invited submission to Foundations and Trends in Electric Energy Systems, 2018.

[23] S. Bolognani and S. Zampieri, "On the existence and linear approximation of the power flow solution in power distribution networks," IEEE Trans. Power Syst., vol. 31, no. 1, pp. 163-172, Jan. 2016.

[24] D. Mehta, D. K. Molzahn, and K. Turitsyn, "Recent advances in computational methods for the power flow equations," in American Control Conf. (ACC), July 2016, pp. 1753-1765.

[25] C. Wang, A. Bernstein, J. Y. Le Boudec, and M. Paolone, "Explicit conditions on existence and uniqueness of load-flow solutions in distribution networks," IEEE Trans. Smart Grid, vol. 9, no. 2, pp. 953-962, Mar. 2018.

[26] D. Lee, H. D. Nguyen, K. Dvijotham, and K. Turitsyn, "Convex restriction of power flow feasibility set," arXiv:1803.00818, Mar. 2018.

[27] D. Bienstock and A. Verma, "Strong NP-hardness of AC power flows feasibility," arXiv:1512.07315, Dec. 2015.

[28] K. Lehmann, A. Grastien, and P. Van Hentenryck, "AC-feasibility on tree networks is NP-hard," IEEE Trans. Power Syst., vol. 31, no. 1, pp. 798-801, Jan. 2016.

[29] D. K. Molzahn and I. Hiskens, "Sparsity-exploiting moment-based relaxations of the optimal power flow problem," IEEE Trans. Power Syst., vol. 30, no. 6, pp. 3168-3180, Nov. 2015.

[30] C. Josz and D. K. Molzahn, "Lasserre hierarchy for large scale polynomial optimization in real and complex variables," SIAM J. Optimiz., vol. 28, no. 2, pp. 1017-1048, 2018.

[31] B. Kocuk, S. S. Dey, and X. A. Sun, "Strong SOCP relaxations of the optimal power flow problem," Oper. Res., vol. 64, no. 6, pp. 1177-1196, 2016.

[32] C. Chen, A. Atamtürk, and S. S. Oren, "Bound tightening for the alternating current optimal power flow problem," IEEE Trans. Power Syst., vol. 31, no. 5, pp. 3729-3736, Sept. 2016.

[33] D. K. Molzahn and L. A. Roald, "Towards an AC optimal power flow algorithm with robust feasibility guarantees," in 20th Power Syst. Comput. Conf. (PSCC), June 2018.

[34] J. Lofberg, "YALMIP: A toolbox for modeling and optimization in MATLAB," in IEEE Int. Symp. Comput. Aided Control Syst. Des., 2004, pp. 284-289.

[35] M. R. Narimani, D. K. Molzahn, and M. L. Crow, "Improving QC relaxations of OPF problems via voltage magnitude difference constraints and envelopes for trilinear monomials," in 20th Power Syst. Comput. Conf. (PSCC), June 2018. 